\title{
Germanica
}

\section{Theodor Plievier, romancier-reporter des deux guerres mondiales}

Theodor Plieviers Zeitromane der beiden Weltkriege

\section{Pierre Vaydat}

\section{(2) OpenEdition}

12 Journals

Édition électronique

URL : http://journals.openedition.org/germanica/2242

DOI : $10.4000 /$ germanica.2242

ISSN : 2107-0784

Éditeur

Université de Lille

\section{Édition imprimée}

Date de publication : 30 juin 2001

Pagination : 105-118

ISBN : 9782913857056

ISSN : 0984-2632

\section{Référence électronique}

Pierre Vaydat, «Theodor Plievier, romancier-reporter des deux guerres mondiales », Germanica [En ligne], 28 | 2001, mis en ligne le 07 octobre 2013, consulté le 06 octobre 2020. URL : http:// journals.openedition.org/germanica/2242 ; DOI : https://doi.org/10.4000/germanica.2242 


\section{Theodor Plievier, romancier- reporter des deux guerres mondiales}

Theodor Plieviers Zeitromane der beiden Weltkriege

Pierre Vaydat

1 Th. Plievier a consacré aux deux guerres mondiales l'essentiel de sa production romanesque. Des Kaisers Kulis et Der Kaiser ging, die Generäle blieben, publiés en 1930 et en 1932 respectivement, font revivre des épisodes de la Grande Guerre et leurs séquelles politiques immédiates. Ce sont des romans qu'on peut qualifier d'historiques, puisqu'ils relatent des faits appartenant à une époque révolue bien que récente; néanmoins, les procédés utilisés sont ceux du roman d'actualité : technique narrative unanimiste, découpage en scènes courtes, où la part des dialogues est prépondérante; descriptions très documentées, alliant la concision et la précision du détail technique. Le mode de représentation est celui du reportage, privilégiant les impressions instantanées, réduisant au minimum la distance entre les événements et les personnages impliqués. Des Kaisers Kulis raconte la bataille du Jutland (Skagerrak-Schlacht), la mutinerie de 1917, puis, dans un dernier chapitre, la révolte décisive des marins de Kiel lorsque leurs officiers ordonnent l'appareillage pour provoquer, alors que sont déjà engagés les pourparlers d'armistice (et donc pour les saboter dans un paroxysme de désespoir nationaliste), un ultime affrontement suicidaire avec la flotte britannique. Der Kaiser ging, die Generäle blieben débute sur le front de l'Ouest, à la fin de l'été 1918, au moment où la défaite des armées allemandes est imminente. Les scènes de guerre n'occupent ici que les premières pages : le reste du livre décrit la « révolution de novembre ", toujours en style de reportage (emploi constant du présent de narration, rythme haletant du récit, commentaires brefs des moments importants de l'action). L'échec de la révolution est consommé à la page finale, où l'on voit Ebert s'assurer l'appui du général Groener et de la caste militaire pour empêcher la prise du pouvoir par une extrêmegauche qui le terrifie parce qu'elle menace de plonger l'Allemagne dans le chaos d'un pays bolchevisé. 
2 Douze années plus tard, en 1943-1944, Plievier, réfugié en URSS, écrit son premier roman sur la Deuxième Guerre mondiale, le célèbre Stalingrad, dont l'écriture emploie les mêmes techniques narratives, celles de la "Nouvelle Objectivité ", mais avec une tendance plus marquée encore que dans Des Kaisers Kulis à utiliser parallèlement le grandissement expressionniste, ce que justifie le caractère à l'évidence apocalyptique du sujet. De retour en Allemagne, Plievier gagne en 1947 la zone américaine. Il écrit alors Moskau (1952) et Berlin (1954), construisant une trilogie romanesque de la guerre sur le front de l'Est.

3 Ces romans, qu'ils portent sur l'un ou l'autre conflit, ont un thème commun : la faillite de l'humanisme socialiste. Plievier peint d'immenses fresques qui font partager au lecteur, passionné par la narration-reportage, le devenir de tout un peuple : le destin catastrophique de l'Allemagne, puis de l'Europe entière, dont est responsable le militarisme prussien à partir du moment où il s'est allié d'abord, sous Guillaume II, à l'impérialisme pangermaniste, puis, sous la République de Weimar, au nationalisme raciste, finissant par pactiser avec les hitlériens dont il se fait l'instrument, étant donné la convergence du projet de nation soldatique forgé par Ludendorff et Ernst Jünger et des plans nazis d'hégémonie raciste sur le continent européen jusqu'à l'Oural.

Le terme de reportage ne doit pas être pris dans une acception péjorative. Plievier ne choisit pas un moyen médiatique avant la lettre de créer sans peine une impression d'authenticité factuelle. En faisant entrer de plain-pied le lecteur allemand dans la lugubre histoire récente de sa nation, il ambitionne d'obtenir ce que nous appelons aujourd'hui un travail de mémoire. Le récit doit l'inciter, par la gradation des effets de choc, à rentrer en lui-même pour mesurer sa part de culpabilité, sa participation active ou passive aux errements, puis aux crimes collectifs.

Pendant la Grande Guerre, Plievier a servi comme matelot à des postes exposés. Cependant, il a choisi dès son premier livre de ne pas transcrire un vécu personnel forcément limité, mais de figurer au contraire des événements de vaste ampleur dont il n'a pas été le témoin direct. Le grandiose récit de la bataille du Jutland dans Des Kaisers Kulis est probablement l'une des descriptions les plus impressionnantes que l'on puisse lire sur un affrontement naval dans toutes les littératures. C'est un chapitre soigneusement composé, basé sur une étude attentive des documents disponibles, car Plievier n'avait pas participé à l'action. Harry Wilde, son secrétaire, raconte qu'il est allé emprunter à la Preussische Staatsbibliothek "alle nur erdenklichen Bücher über die deutsche Hochseeflotte und die Erlebnisse einzelner Matrosen $»^{1}$. Il s'agit d'une reconstitution naturaliste, semblable de ce point de vue à celle de la bataille de Verdun dans Les Hommes de bonne volonté de Jules Romains, qui est au demeurant postérieure (Prélude à Verdun et Verdun ont paru en 1938); avec toutefois cette différence que Plievier a fait personnellement l'expérience prolongée de la peur, de la promiscuité et de l'épuisement, tandis que l'écrivain français, n'ayant pas été mobilisé, ne peut en parler que par procuration ${ }^{2}$. L'écart entre les méthodes des deux auteurs est cependant moins grand qu'il n'y paraît. C'est ainsi que Plievier, bien qu'il n'ait jamais été fantassin, réussit cependant au début de son roman suivant (Der Kaiser ging, die Generäle blieben) à faire vivre au lecteur les minutes ultimes de la vie d'un soldat qu'un tank ennemi va écraser. De même, bien qu'il n'ait jamais eu de formation militaire supérieure, Plievier sait décrire dans les pages suivantes de ce livre l'activité d'un lieutenant à l'arrière immédiat de la ligne de front, celle d'un aide de camp, puis restituer de façon plausible les réflexions de Ludendorff dans le train spécial qui 
l'emmène vers Berlin où il va exiger du nouveau gouvernement hâtivement constitué sur la base d'une majorité parlementaire qu'il lui soit fourni à tout prix des renforts. Là encore, Plievier s'est livré à un intense travail de documentation: le «Quellennachweis» figurant en appendice de Der Kaiser ging... contient une cinquantaine de titres, auxquels s'ajoute une liste des collections de journaux consultées.

6 Pour écrire ses trois grands romans sur le conflit germano-russe de 1941-1945, Plievier va se trouver dans un rapport similaire à l'événement. Stalingrad a certes été écrit en URSS presque aussitôt après la bataille, mais l'auteur n'y a pas assisté, ayant été évacué par les autorités soviétiques à Tachkent, puis à Oufa.

7 La genèse de Stalingrad est connue. Chargé par les Soviétiques d'analyser les lettres saisies sur les Allemands tués ou prisonniers, afin de fournir des idées à une action psychologique un peu moins fruste et inefficace que la propagande sommairement marxiste déployée jusque-là contre l'envahisseur hitlérien, Plievier décide d'exploiter la masse de témoignages dont il dispose pour composer une œuvre fictionnelle d'actualité. Son but est de conférer à l'écrasante défaite subie par la Wehrmacht un sens moral exemplaire, susceptible de contrecarrer l'influence de la propagande monumentalisante immédiatement mise en œuvre par les nazis.

8 Une méthode analogue a été employée pour la rédaction des romans Moskau et Berlin, formant le premier et le troisième volet du triptyque. Plievier a recouru aux souvenirs du comte Thilo Werthern, qui avait servi comme officier dans les chars lors de l'avance sur Moscou. Pour décrire la débâcle du côté russe durant l'été 41, Plievier a utilisé des informations que lui a fournies Valerian Lebedev, ex-lieutenant de l'Armée Rouge. Plievier a procédé de même pour élaborer Berlin. N'ayant quitté l'URSS que le 15 mai 1945, il n'a pu être témoin de l'encerclement et de la prise d'assaut de la capitale du Reich. D'après H. Wilde, il a commencé à rassembler la documentation nécessaire à la rédaction du livre dès $1948^{3}$, mais son biographe ne donne guère de détails concernant une phase préparatoire qui s'est étendue sans doute sur de longues années. (Il manque à ce jour une étude d'ensemble sur les sources de la trilogie. Dans la postface qu'il a rédigée pour la réédition du roman, Hans-Harald Müller signale l'existence d'un «Plievier-Nachlass $»^{4}$ ).

9 Si l'unité thématique et le recours constant aux mêmes procédés narratifs sont faciles à établir, la cohérence idéologique est beaucoup moins évidente.

Plievier a été sans conteste un homme de gauche, si l'on entend par ce terme la résolution de défendre des positions généreuses en faveur des humiliés et des opprimés. Mais il n'a jamais pu ou voulu renoncer à son indépendance d'esprit pour se soumettre à une discipline de parti. Il n'a été membre ni du KPD, ni du SPD. Bien qu'il se soit rangé d'instinct du côté des marins révolutionnaires, son attitude à l'égard des spartakistes et de l'USPD est demeurée réservée. Dans Der Kaiser ging..., il dénonce la tactique des socialistes majoritaires qui veulent entraver le déroulement d'une révolution non souhaitée (voir le récit de la visite de Noske à Kiel), ainsi que l'entêtement de Ebert à préserver coûte que coûte l'appareil du SPD, quitte à s'allier aux militaristes; mais il laisse entrevoir que l'extrême-gauche n'a pas de programme dépassant le niveau du slogan et que la capacité organisationnelle de révolutionnaires authentiques lui fait défaut. Quant aux socialistes indépendants (USPD), il les montre hésitants, bavards et divisés. 
11 Il est encore plus difficile d'assigner à Plievier une appartenance politique définissable quand on examine la trilogie de la Deuxième Guerre mondiale. Ce qui lui donne son unité, c'est bien sûr sa matière et le retour de quelques personnages principaux. Mais, du point de vue idéologique, le contraste est frappant entre Stalingrad et les deux autres volumes. Stalingrad ne contient aucune critique du système soviétique et incrimine un militarisme prusso-allemand présenté plutôt comme égaré par la séduction pangermaniste que comme fondamentalement malfaisant. Plievier met surtout en relief le sacrifice inutile de la meilleure substance allemande (la $6^{\mathrm{e}}$ Armée de Paulus est une armée d'élite, ses soldats exceptionnellement robustes proviennent pour une large part de la paysannerie), tout en évoquant en arrière-plan les souffrances endurées par les populations civiles de l'URSS. Cette orientation s'explique lorsqu'on sait que Plievier est proche à ce moment des thèses de propagande du Comité National de l'Allemagne Libre (Nationalkomitee Freies Deutschland). La scène finale grandiloquente de Stalingrad, où l'on voit Vilshofen, promu général pour ses exploits à la tête des blindés qui ont pris la ville, et l'indestructible sous-officier August Gnotke marcher côte à côte vers une captivité censément rédemptrice en est l'illustration plutôt naïve : un exemple typique (rare tout de même chez Plievier) de mauvaise littérature à base de bons sentiments, conjoncturels mais néanmoins visiblement sincères. Moskau et Berlin, écrits en Allemagne de l'Ouest au moment où la RFA rejoignait le Pacte Atlantique, sont en revanche les constats d'échec d'un marxisme-léninisme auquel Plievier n'avait d'ailleurs jamais adhéré. L'oppression totalitaire, jointe à une brutale incompétence, annihile le rêve de justice, essence du socialisme, les communistes idéalistes (le général Narychkine, le capitaine Ouralov) sont les victimes désignées du système. Celui-ci n'a pu survivre que grâce à l'implacable volonté nazie d'asservissement et d'extermination, qui ne laissait pas d'autre choix aux peuples de l'URSS, après les semaines initiales de débâcle, que le durcissement désespéré de la résistance et la contre-offensive au prix d'effroyables pertes.

Ces changements d'optique ont été interprétés comme une provocation: pour les communistes, Plievier est un traître. Ce revirement ne doit pas faire oublier pourtant la prédominance des éléments de continuité.

Il y a d'abord la littérarité même de cette entreprise romanesque qui couvre plus de trente années de cataclysmes historiques : la théâtralisation de l'horreur spécifique de la guerre industrialisée, mais aussi de la volonté de puissance qui en est la cause principale. Elle est visible par exemple dans le "dénombrement des vaisseaux » qui vont livrer la bataille du Jutland (cf. le chapitre "Skagerrak» dans Des Kaisers Kulis), comme dans le monologue intérieur du pilote de l'avion de reconnaissance survolant l'espace russe juste après le déclenchement de l'invasion allemande, le 22 juin 41 . L'esthétisation de la guerre contraste de façon déroutante avec l'intention pacifiste. C'est l'attraction irrésistible de la guerre-spectacle, indissociable de la mise en récit ${ }^{5}$. La littérature de gauche l'a employée comme vecteur des énergies morales tout autant que celle de droite. Il suffit de se rappeler la description de la bataille de Valmy par Michelet ou les pages fulgurantes de L'Espoir d'André Malraux.

Une littérature de guerre qui renoncerait délibérément à produire un souffle d'épopée entraînant le lecteur à se mettre au diapason de l'exaltation des combattants s'interdirait d'autres procédés que la dérision ou le grotesque. Une écriture de cette sorte serait justifiable du point de vue humanitaire, mais se limiterait à un banal 
constat de folie collective : c'est l'optique pour laquelle a opté Günter Grass lorsqu'il fait évoquer le déroulement des hostilités par le nain Oskar dans Die Blechtrommel.

Chez Plievier, l'esthétisation de la guerre est tout aussi présente que chez Ernst Jünger, figure emblématique de l'autre bord. Mais elle est aux antipodes d'une mystique de la violence régénératrice. Plievier recherche un effet de dégrisement par la peinture de la mort massifiée. Il reste qu'il n'était point exempt d'une vanité d'artiste. Il donnait volontiers lecture des pages sur la bataille du Jutland, dont il était particulièrement fier ${ }^{6}$. Les scènes de bataille dans Stalingrad, Moskau et Berlin ont la même vigueur épique. Sous cet angle, Plievier peut soutenir la comparaison avec Tolstoï. Mais il est bien sûr inférieur à lui pour ce qui touche le génie essentiel du romancier, qui est de camper des personnages inoubliables. Sa psychologie est cursive, typique du roman d'actualité ; elle fait ressortir presque exclusivement la représentativité sociologique des comportements individuels. Lorsque Plievier imagine les figures hors du commun qui lui servent de porte-parole, Vilshofen et Gnotke, il les surcharge d'un symbolisme qui sent l'artifice et leur confère une forme d'intelligence critique en parfaite contradiction avec leurs antécédents.

La thématique des romans de la Grande Guerre était l'échec de la révolution socialiste en Allemagne, qui a permis au militarisme d'y reprendre force et vigueur.

Ce réquisitoire contre le militarisme prusso-allemand s'amplifie dans le triptyque de la Deuxième Guerre mondiale. Sur le front russe, l'atrocité traditionnelle de la guerre non seulement s'amplifie, mais change de nature. Plievier a posé en termes nets, et cela en pleine époque adenauérienne, la question impopulaire en RFA de la culpabilité collective allemande.

18 Une deuxième caractéristique de la trilogie est la place dévolue à la peinture de la réalité stalinienne. C'est encore une fois le problème de l'échec du socialisme dès qu'il est géré par un appareil. La faute du socialisme allemand avait été sa funeste tendance au compromis avec les couches sociales conservatrices. Le communisme russe, à l'inverse, s'est décidé à mettre en place un appareil répressif impitoyable, qui, très rapidement, n'a plus eu comme but que sa préservation devenue fin en soi. Au point de vue des convictions philosophiques, Plievier reste un anarchiste, marqué par la lecture de Stirner. Il débusque l'autoritarisme dans toutes les formes de pouvoir, mais ne trouve à leur opposer, en fin de compte, une fois dégoûté du communisme, qu'un humanitarisme chrétien diffus dont l'auteur de «L'Unique » aurait ricané. Plievier n'a pas de message programmatique à proprement parler. Faut-il le reprocher à un écrivain? Tolstoï, après tout, ne proposait qu'une sagesse rapetissant l'Histoire, alors que son génie de prosateur la faisait revivre avec un relief sans pareil.

Cela dit, il y a bien dans l'œuvre de Plievier une préoccupation politique centrale : la dénonciation $\mathrm{du}$ militarisme prusso-allemand, présenté comme une tradition d'obéissance inconditionnelle, de volontarisme impitoyable et de nationalisme prédateur.

20 Dans Des Kaisers Kulis, les griefs restaient, comme le voulait d'ailleurs la conception même du roman, où les événements sont rapportés, sauf exception, tels que les percevaient les équipages, ceux du quotidien: il est surtout question de la morgue tyrannique des officiers, de leurs privilèges en matière notamment de nourriture, alors que les matelots sont sous-alimentés. Les mots-clef sont "Schufterei », "Willkür » et «Kohldampfschieben». Les officiers veulent la prolongation de la guerre, car ils espèrent le "Siegfrieden ", la réalisation par une victoire éclatante des objectifs 
annexionnistes. La tension dramatique nait de l'irritation croissante des équipages las d'une oppression monotone et rendus furieux par le mépris des supérieurs. Le pouvoir militaire réussit encore à mater la révolte de 1917, mais il cède d'un coup lorsqu'il est privé de sa légitimité monarchique fin octobre 18.

21 La perspective s'élargit dans Der Kaiser ging... Elle est devenue celle du reporter omniprésent, gagnant du coup en puissance analytique. Cette fois, le réquisitoire est centré sur l'utopie de la nation intégralement militarisée, but déclaré de Ludendorff (voir le chapitre initial «Die Macht»). Pour survivre à la défaite, la caste militaire se désolidarisera du pouvoir impérial discrédité et conclura un pacte avec le SPD, auquel la lie depuis août 14 une longue connivence de fait en dépit des oppositions doctrinales.

Contrairement aux deux premiers ouvrages, qui s'adressaient à un public de gauche au sens large, Stalingrad est destiné en priorité à un groupe cible tout autre et bien identifiable : la nouvelle classe militaire qu'est le corps des officiers de la Wehrmacht. Il est par conséquent, à l'inverse des autres romans, porteur d'un message précis. Les conditions extrêmes dans lesquelles se déroule la campagne de Russie ont renforcé l'unité de cette strate, formée pourtant de deux éléments antagoniques: les traditionalistes prussiens ou prussianisés d'un côté, de l'autre les nazis de la jeune génération. Ce qui les rendait solidaires, outre la camaraderie forgée par la vie du front, c'était la vision d'avenir du pangermanisme continental. L' "Idée » du "Grossraum » date d'avant la Première Guerre mondiale; elle a inspiré le féroce traité de BrestLitovsk. L'anéantissement de la $6^{\mathrm{e}}$ Armée, avec son cortège de souffrances hideuses, est le châtiment infligé par la Providence au prussianisme. Pourtant, celui-ci n'est pas exclu de toute rédemption. Il peut encore la mériter s'il prend conscience du crime collectif auquel il a prêté son concours. Si les généraux de Stalingrad assiégé qui, après avoir fait tuer leurs hommes sans remords (autant par la faim, le froid et la dysenterie que par le feu ennemi) pour obéir à l'ordre de Hitler, ont ensuite refusé de se suicider, leur décision de continuer à vivre n'aura une signification morale que s'ils prennent la résolution de combattre le nazisme à visage découvert. Plievier exige des militaires qu'ils fassent publiquement œuvre de repentance en dressant la Wehrmacht contre Hitler.

Plievier s'est détaché intérieurement très vite du NKFD, qui n'a connu au demeurant qu'une brève existence (juillet 43-novembre 45). Il y a lieu d'observer toutefois que le message de Stalingrad ne fait pas apparaître d'allégeance à l'idéologie marxiste et qu'il relève plutôt d'un christianisme sécularisé, dans lequel sont présentes les notions de péché et d'expiation. Il n'y a pas lieu, en tout cas, de parler d'adaptation opportuniste à une éventuelle idéologie de rechange de certains militaires: la même conviction se retrouvera dans Moskau et Berlin.

24 Moskau condamne la réalité soviétique tant du point de vue éthique que pragmatique. Mais, chose rare dans le corpus des romans de guerre de l'époque adenauérienne ${ }^{7}$, Plievier parle en termes clairs des massacres de Juifs, des exécutions sommaires de commissaires soviétiques aux armées et de prisonniers désignés arbitrairement pour être abattus en représailles lorsque des colonnes de captifs passent devant un cadavre allemand. Notons cependant que le crime collectif, dans Moskau, est imputé aux SS, aux SD et aux fonctionnaires du «Generalgouvernement » de la Pologne occupée, ce qui va dans le sens du discours généralement tenu à l'époque en Allemagne de l'Ouest sur la Wehrmacht victime de fait d'une tyrannie abhorrée. Il fait dire au colonel Zecke (qui sera également le porte-parole de ses idées dans Berlin) : 
Guderian allein gegen Hitler, das ist zu ungleich. Guderian ist ein ausgezeichneter Truppenoffizier, ein Meister grosser Bewegungen, hat grosse Konzeptionen, beherrscht die moderne Panzertaktik souverän, doch darüber hinaus geht seine Phantasie nicht. Er ist ausserstande, einen Mann wie Hitler zu begreifen, er sieht ihn, als ob er ein Wilhelm II. oder ein Wilhelm III. wäre und klammerte dabei die ganze Einmaligkeit Hitlers, die zeitgebundene Problematik und die Dämonie seines Auftretens aus!

In der politischen Konzeption noch ein Weltkriegsoffizier wie viele andere. Auch unser Bomelbürg sagt sich : Ich muß mich doch darauf verlassen können, dass der Staat in Ordnung ist! Der Inhalt ist nicht seine Sache, geht ihn nichts an, darum bekümmert er sich nicht!

Ja, das ist wohl unsere Tragik: von Politik verstehen wir nichts, doch wenn die Aussenpolitik eine Katastrophe herbeibeschwört, dann sollen plötzlich die Waffen das Ergebnis dieser falschen Aussenpolitik wieder in Ordnung brigen, und das geht nicht, das stellt sich immer wieder heraus. Im ersten Weltkrieg ist das Heer überfordert worden, und wir dürfen nur hoffen, dass es diesmal nicht wieder so sein wird.

Dans un passage situé vers la fin du livre, Plievier va plus loin. Il relate comment Hitler, après l'échec de l'offensive allemande devant Moscou, relève de leurs fonctions les généraux les plus célèbres et les plus compétents. Il déplore cette « saignée » et suggère que c'est seulement à partir du moment où ces officiers supérieurs prestigieux ont été écartés que la guerre à l'Est s'est véritablement déshumanisée. Il y a plus surprenant encore. Plievier laisse entendre que cette purge prive l'Europe centrale d'une source indispensable de pouvoir civilisé :

Das war der Aderlass an der höchsten militärischen Führung Deutschlands; und mehr - es bedeutete die Ersetzung des selbständigen Denkens durch die Schablone und in der Konsequenz die Austrocknung des organisatorischen und moralischen Vermögens des deutschen Volkes; und noch mehr - die Austrocknung einer bedeutenden Kraftquelle in der traditionellen Mitte des Kontinents, bedeutete den Einzug der Steppe in Europa. Tausendfünfhundert bis zweitausend Generalstabsoffiziere waren betroffen: Kommandeure, die ihre militärische Einsicht über den Parteibefehl stellten, die nicht in einer befohlenen Weltanschauung, sondern im eigenen Gewissen die höchste entscheidende Instanz erblickten, die internationale Abmachungen respektiert haben wollten und auch aus diesem Kriege mit "weisser Weste» herauszukommen trachteten. Treu und Glauben, Anständigkeit und Ritterlichkeit blieben auf der Strecke. Das Parallelogramm der sittlichen Kräfte auf den östlichen Schlachtfeldern war gespannt, das Gesicht der deutsch-russischen Front endgültig geprägt. Es konnte sich nur noch um Vernichtung handeln.

Il convient de mettre cette présentation disculpante en relation avec le soutien de Plievier au réarmement allemand ${ }^{8}$. Moskau n'a pas manqué de susciter la colère des communistes français ${ }^{9}$. Certes, G. Badia avait tort de se gausser de la critique virulente du régime stalinien, mais il pouvait affirmer non sans vraisemblance : «En réhabilitant la Wehrmacht, (Plievier) apporte de l'eau au moulin de la politique adenauérienne. »

L'anarchisme antimilitariste des années 30 a donc fait place, serait-on tenté de dire, au conformisme atlantiste. G. Badia, dans l'article cité, a franchi le pas, en soutenant que l'auteur de Moskau était «devenu officiellement un agent des services américains d'occupation en Allemagne ». En revanche, Plievier estimait être demeuré fidèle à ses convictions humanistes de toujours. Il se réclamait en toute bonne foi des Dix Commandements et de la Charte de l'Atlantique. Contre ses détracteurs, qui l'accusaient de soumission intéressée aux Américains, il faisait valoir qu'on ne pouvait l'accuser d'avoir tourné casaque parce qu'il dénonçait le totalitarisme soviétique ${ }^{10}$. 
Rudolf Hagelstange l'en approuvait dans un texte rédigé pour une revue française où s'exprimaient les opinions de la gauche libérale ${ }^{11}:$ «S'il fut autrefois révolutionnaire, c'était beaucoup moins pour défendre une classe que les droits de l'homme en général, en faveur desquels sa classe - jadis - avait combattu. [...] Si, en tant qu'ancien hôte de l'Union Soviétique, il s'est abstenu de traîner dans la boue ceux qui l'avaient hébergé pendant de longues années, il a pris en tant que technicien politique (sic !) une position sans équivoque. Plievier fut dès lors (sic) l'adversaire du bolchévisme au point de refuser tout compromis. Nul ne peut mettre en doute qu'il n'ait été aussi un adversaire de la guerre [...] Mais (ce que certains voudraient cacher à leurs yeux et à ceux des autres) il savait que l'amour de la paix n'est pas une protection contre l'impérialisme communiste, cette force qui prend dans ses pièges une victime après l'autre et que la faiblesse rend non pas plus tolérante, mais plus agressive. Il aurait fallu qu'il cessât de penser par lui-même pour se dissimuler les périls auxquels ses divisions et son absence de protection exposent le monde encore "libre". Il était de ceux qui avaient perdu une révolution. Il ne voulait à aucun prix être de ceux qui perdaient leurs dernières libertés, leurs ultimes droits. [...] Plievier fut durablement "l'enfant perdu d'une révolution manquée"; il le fut de la petite révolution qu'il avait lui-même manquée (sic) à Kiel, et aussi de la grande, que le prolétariat a perdue, peut-être définitivement, dans les semaines où les marins de Cronstadt furent balayés par les batteries trotzkystes. »

Cette année 1956 voyait paraître Berlin, l'achèvement du triptyque. Dans cette œuvre, nous voyons revenir le colonel Zecke, qui avait joué dans Moskau un rôle épisodique, mais s'y distinguait déjà néanmoins par ses facultés d'analyse. Comme Vilshofen, Zecke s'est rallié à l'humanisme. Il incarne le type de l'officier allemand revenu de ses erreurs, capable de réflexion autonome, et utilisable par conséquent dans la lutte que l'Occident doit mener contre le totalitarisme soviétique, après avoir vaincu le totalitarisme nazi.

Force est de reconnaître que Berlin se rapproche davantage encore que Moskau de l'état général des esprits dans la RFA d'Adenauer. Plievier flétrit le comportement des troupes d'assaut soviétiques (exécutions sommaires et viols tacitement ou même ouvertement encouragés par leurs chefs). Il condamne aussi le bombardement de Dresde, affirmant qu'il a été ordonné par Churchill et Roosevelt pour complaire à Staline. Mais en même temps la voix intérieure de Zecke lui murmure que ces horreurs sont une réplique à la décision nazie, engageant le peuple allemand dans son ensemble, d'ériger en doctrine d'État le racisme génocidaire. D'autre part, Plievier-Zecke, s'il déplore l'impéritie des conjurés du 20 juillet 44 et critique le conservatisme trop manifeste de leur programme de gouvernement, voit en eux, comme dans les autres résistants, "ouvriers, étudiants, femmes ", ceux que Dieu a élus et dont les noms sanctifieront une "Allemagne du recommencement» («wenn Deutschland sich zu neuem Beginn erhebt »).

De 1918 à 1954, Plievier a donc vécu l'effroyable désillusion qui a imprimé au xxe siècle sa marque distinctive. La faillite morale des révolutions socialiste (en Allemagne) et communiste (en Russie et dans les pays satellites) lui a fait redécouvrir un patriotisme longtemps discrédité à ses yeux par la déviance pangermaniste, mais ce retour à ses racines allemandes ne l'a pas amené pour autant à renier ses convictions humanitaires et cosmopolites de toujours. Le patriotisme allemand surgissait à la fin de Stalingrad; dans Berlin, il imprègne presque chaque page et sa formulation prend l'allure très souvent d'une profession de foi. 
31 Non que Plievier tente de disculper l'Allemagne. Il persiste à reconnaître la responsabilité que portaient les ambitions conquérantes, la morgue et l'inhumanité foncière de ses dirigeants et de ses élites dans le déclenchement des deux Guerres Mondiales. Il a cessé de croire, qui plus est - si tant est qu'il y ait jamais cru - à la possibilité de racheter un passé d'infamie par l'avènement d'une nouvelle Internationale à l'édification de laquelle - selon la doctrine des fondateurs de la RDA les masses populaires allemandes, une fois désaliénées par l'éducation marxiste, prendraient une part glorieuse. La rédemption par une Histoire progressant de façon dialectique est un poncif dont on ne relève aucune trace dans son œuvre.

Pour le peuple allemand, tel est le message final de Plievier, le salut ne peut venir que de l'adhésion - sincère et non tactique - aux valeurs de liberté qui sont, affirme-t-il, la substance de la civilisation occidentale. Les considérations sur l'avenir de la civilisation européenne que Plievier a développées dans son discours de mai 1948 nous semblent aujourd'hui moins utopiques et même moins superficielles que lorsqu'il les a exposées en plein début de la guerre froide :

Individuen und Völker und zuerst die Völker des europäischen Kulturkreises stehen dem Entweder-Oder gegenüber. Entweder Handeln oder Untergehen! Und Handeln heisst in diesem Falle: Seelische Neugeburt des Individuums! Gesellschaftliche Neugeburt von Völkern! Neugestaltung der politischen Beziehungen der Völker untereinander! Und noch genauer: Neugestaltung unter Aufgabe enger nationalegoistischer Interessen und unter Voranstellung der allen gemeinsamen und das Ganze fördernden Interessen. Und Handeln bedeutet nicht umstürzen, es bedeutet Erfüllen.

Die geistigen Grundlagen sind uns gegeben. Und wenn wir das uns Gegebene beginnend mit den zehn Geboten vom Berge Sinai und endend mit den Thesen der Atlantic-Charta - von den Fesseln des Papiers befreien und alle sittlichen Gebote und die gegebenen bürgerlichen Freiheiten und Rechte des Menschen in das tägliche Leben und die alltäglichen Beziehungen der Menschen untereinander übertragen, und wenn Intoleranz und Rassenwahn und Sklaverei nicht nur auf dem Papier, sondern in der Wirklichkeit beseitigt sein werden, wenn alle uns gegebenen sittlichen Prinzipien nicht nur Angelegenheiten feierlicher Staatsakte und Inhalte kirchlicher Sonntagspredigten, sondern Gesetze auch auf den Gebieten der Gesellschaft und Wirtschaft geworden sind, dann haben wir das Revolutionärste vollbracht, das Menschen überhaupt zu vollbringen vermögen, und haben doch nur erfüllt, was die Menschheit von weit her begleitet und bis hierher zusammengehalten und lebend erhalten hat ${ }^{12}$

Mais l'adhésion à l'Occident suppose le repentir. Si le personnage de August Gnotke est le seul à reparaitre dans tous les volumes de la trilogie, c'est parce qu'il a pour fonction d'incarner cette exigence. Gnotke symbolise beaucoup de choses à la fois, trop peutêtre pour être un personnage crédible: la vitalité, les qualités de fiabilité et de compétence technique de l'Allemand, mais aussi sa propension à l'égarement, puis, finalement, son aptitude à une conversion authentique, qui témoigne de sa moralité au fond inaltérable.

August Gnotke est un ancien membre des SA. Il s'en est détaché par écœurement après la Nuit des Longs Couteaux. Sous-officier modèle de la Wehrmacht, il est arrêté, dégradé, puis envoyé dans une compagnie disciplinaire pour avoir tenu des discours hostiles à Hitler. Ayant survécu à ces épreuves, il est fait prisonnier par les Russes après la capitulation de la $6^{\text {e }}$ Armée. Mais la captivité en URSS n'est pas ce que laissait entrevoir l'auteur à la dernière page de Stalingrad, elle est très loin d'être un moyen de purification morale : car le régime soviétique - telle est la thèse de Berlin - est une "Église sans la Grâce». Après avoir subi un lavage de cerveau qui le transforme 
psychiquement en automate sans toutefois lui faire perdre ses qualités d'adaptation et son incroyable endurance, Gnotke devient un agent que les services de renseignement soviétiques infiltrent dans les lignes allemandes. Revenant toujours indemne des missions les plus dangereuses, il devient après la victoire sur le $\mathrm{III}^{\mathrm{e}}$ Reich domestique chez le Premier Ministre de Thuringe, qu'il est naturellement chargé d'espionner. Il accomplira scrupuleusement cette besogne, mais, indigné soudainement par la répression du soulèvement populaire du 17 juin 1953, il se fera tuer exprès à Berlin dans les rangs des insurgés.

La mort de Gnotke renouvelle le martyre du marin Alwin Köbis, l'une des figures principales dans Des Kaisers Kulis (personnage réel, à la différence de Gnotke), fusillé en septembre 17 pour rébellion et discours subversifs.

L'itinéraire de Th. Plievier aboutit à un idéal sacrificiel qui, en réalité, avait déjà une présence, certes beaucoup plus discrète, dans son premier roman. L'abnégation comme vertu essentielle, parce qu'elle est féconde même lorsqu'elle semble rester vaine dans l'immédiat au plan du " change of heart » chez ceux qui sont censés être des citoyens : tel est l'enseignement initial, mais aussi l'enseignement ultime dispensé par une œuvre qui veut témoigner non seulement des horreurs de la guerre, mais également de leur majoration par la pire perversion du politique. Indéniablement, il y a chez notre auteur un retour du religieux. S'agit-il d'une réaction au désenchantement idéologique ? C'est une hypothèse vraisemblable, mais il est tentant aussi de ne pas écarter un autre aspect de la question. Plievier s'aligne, délibérément ou inconsciemment, sur le christianisme supra-confessionnel qui se généralise à l'époque dans le monde anglo-saxon et soustend sa mentalité démocratique. Ainsi, la régénération de l'Allemagne par le christianisme est un postulat qui rend acceptable à l'opinion d'outre-Manche la plus hostile aux Allemands le pouvoir d'Adenauer et de la $\mathrm{CDU}^{13}$. On sait avec quelle véhémence sarcastique un Heinrich Böll a protesté contre cette manière de faire le pont, dont la suite des événements a néanmoins démontré l'efficacité politique.

L'autre leçon du discours de mai 48 comme de Moskau et de Berlin était que l'Allemagne devait cesser de se vouloir l'instance régénératrice du monde, projet discrédité par l'égoïsme national mégalomane. À sa façon, Plievier n'est pas moins réaliste que le chancelier Adenauer. Du vieux sage de Bonn, il n'a, il est vrai, ni le machiavélisme, ni la mentalité patriarcale. Il partage en revanche la conviction que Adenauer n'a pas formulée explicitement coram populo, à savoir qu'il est également impossible de se débarrasser du poids moral du passé nazi et d'éliminer de la vie publique tous ceux qui ont participé à l'entreprise hitlérienne.

\section{NOTES}

1. Harry Wilde, Theodor Plievier. Nullpunkt der Freiheit, Verlag Kurt Desch, München Wien Basel, 1965, p. 209. 
2. Cf. ma contribution intitulée «Le roman de la Grande Guerre en France et en Allemagne. Esquisse d'une approche comparative ", in J.-J. Pollet et A.-M. Saint-Gille (éd.), Écritures francoallemandes de la Grande Guerre, Artois Presses Université, Arras, 1996, notamment pp. 214-217.

3. Op. cit., 474.

4. Th. Plievier, Berlin, Kiepenheuer und Witsch, Köln, 1985, Nachwort v. H.-H. Müller, p. 761.

5. Cf. Christoph Nickenig, Desastres de la guerra. Studien zum Bild des II. Weltkriegs im Roman, Peter Lang, 1996, p. 21 : «Diese Elemente finden sich auch im Antikriegsroman. »

6. Wilde, op. cit., 225.

7. Cf. Jochen Pfeifer, Der deutsche Kriegsroman 1945-1960. Ein Versuch zur Vermittlung von Literatur und Sozialgeschichte, Scriptor Verlag, Königstein / Taunus, 1981, p. 35-43, 60-61, 112, 147 sq., 160 sq., 190, 197 ; Hans Wagener, "Soldaten zwischen Gehorsam und Gewissen. Kriegsromane und Tagebücher », in : H. Wagener (Hg.), Gegenwartsliteratur und Drittes Reich, Reclam, Stuttgart, 1977 ; Dieter Sevin, Individuum und Staat. Das Bild des Soldaten in der Romantrilogie Theodor Plieviers, Bouvier Verlag Herbert Grundmann, Bonn, 1972, p. 72-73, 85-90.

8. Sur la conférence qu'il a donnée à Paris en novembre 1951: "Die Bundesrepublik und die Wiederbewaffnung ", cf. Wilde, op. cit., 499.

9. Voir G. Badia, « Plievier et von Salomon. Deux faux témoins de leur temps », in : La Pensée, $\mathrm{n}^{\circ} 54$, 1954.

10. Th. Plievier, Einige Bemerkungen über die Bedeutung der Freiheit, Nest-Verlag, Nürnberg, 1948.

11. Preuves, $\mathrm{n}^{\circ} 60$, février 1956.

12. « Einige Bemerkungen... », p. 6-7.

13. Exemple : «My lifelong antipathy to Germany is known, yet for once [...] I acknowledge a German Government and tendency which might perhaps be turned to the account of Christian civilization. " Article de Lord Robert Vansittart dans le Times, mai 1954 ; cité par Norman Rose, Vansittart. Study of a Diplomat, Heinemann, Londres, 1978, p. 283.

\section{RÉSUMÉS}

Dans ses romans de la Première Guerre mondiale, Plievier présente le peuple allemand comme victime de sa caste de maîtres, puis il décrit sa révolte justifiée contre le régime des militaristes et des Junkers. « Les galériens du Kaiser » et «Le Kaiser est parti, mais les généraux sont restés » sont des romans de guerre et d'après-guerre d'une vigueur impressionnante, appartenant à l'évidence à la littérature émancipatrice et même socialiste (au sens large, humanitaire plutôt que strictement politique). Il est moins facile de définir l'esprit de la trilogie romanesque consacrée au front russe durant la Deuxième Guerre mondiale (Moscou, Stalingrad, Berlin). Ici, Plievier montre comment non seulement les élites, mais le peuple lui-même se sont rendu coupables, et leur recommande d'accepter leur défaite en lui donnant un sens expiatoire. De plus, la condamnation de la guerre n'est plus un appel à la révolution; car la deuxième expérience essentielle vécue par Plievier pendant les années 30 et 40 est l'effondrement de l'utopie socialiste et communiste, l'affrontement mortel des totalitarismes nazi et soviétique, mais aussi la parenté profonde unissant au niveau de la praxis les deux régimes de terreur. C'est pourquoi l'anarchiste que fut Plievier se rallie après 1945 à la démocratie occidentale teintée de christianisme et se situe désormais en fait du côté d'Adenauer et des USA, puissance tutélaire. 
In seinen Romanen über den ersten Weltkrieg zeigt Plievier das deutsche Volk als Opfer seiner Herrenschicht und dann in der berechtigten Auflehnung gegen das militaristisch-junkerliche Regiment. Des Kaisers Kulis und Der Kaiser ging, die Generäle blieben sind Kriegs- und Nachkriegsromane von eindringlicher Wucht und gehören zugleich deutlich zur emanzipatorischen, ja zur sozialistischen Literatur, wenn man aus letzterem Begriff die parteipolitische Observanz ausklammert. Viel schwieriger ist es, den Gesinnungsgehalt der Romantrilogie über den II. Weltkrieg an der Ostfront (Moskau, Stalingrad, Berlin) zu bestimmen. Hier schildert Plievier, wie nicht nur die Führungselite, sondern das Volk selbst in Schuld verfielen und empfiehlt ihnen, die Niederlage als Sühne hinzunehmen. Die kriegsgegnerische Botschaft ist überdies nicht mehr der Aufruf zur Revolution, denn Plieviers zweite Haupterfahrung der 30er und 40er Jahre ist der Untergang der sozialistischen und kommunistischen Humanitätsutopie, der todbringende Zusammenstoss des Nazi- und des Sowjettotalitarismus, aber doch auch wieder die Verwandtschaft beider Terrorregime, was die Praxis anbetrifft. So bekennt sich der vormalige Gesinnungsanarchist Plievier nach 1945 zur christlich gefärbten westlichen Demokratie und tritt damit faktisch auf die Seite Adenauers und der amerikanischen Schutzmacht.

\section{AUTEUR}

\section{PIERRE VAYDAT}

Université Charles de Gaulle - Lille III 